\title{
Fiber couplers for silicon-on-insulator photonic IC's with optimized on-chip return loss
}

\author{
Yanlu Li*, Diedrik Vermeulen, Yannick De Koninck, Gunay Yurtsever, \\ Gunther Roelkens, Roel Baets \\ Photonics Research Group, INTEC-department, Ghent University-IMEC \\ Center for Nano- and Biophotonics (NB-Photonics), Ghent University \\ Sint-Pietersnieuwstraat 41,9000, Ghent, Belgium \\ *Yanlu.Li@intec.ugent.be
}

\begin{abstract}
A novel grating coupler design with a high return loss is demonstrated for silicon-on-insulator photonic IC's. The average return loss is around $10 \mathrm{~dB}$ better than that of a commonly used focusing grating coupler.
\end{abstract}

(C) 2011 Optical Society of America

OCIS codes: $050.0050,130.0130$.

\section{Introduction}

Fiber-to-chip grating couplers on silicon-on-insulator (SOI) with high coupling efficiencies have been theoretically and experimentally demonstrated [1]. However, when the grating coupler is used to couple light out of the chip from a waveguide, it can also reflect an amount of light back into the waveguide. This on-chip grating coupler retroreflection should be avoided in many designs, such as circuits containing integrated lasers. In order to suppress the second order diffractive reflection from the grating coupler, the output fiber is often tilted away from the orthogonal direction with a polar angle of $10^{\circ}$ [2]. However, the back reflection can not be totally suppressed by this method, since the effective index mismatch between the slab mode and the grating mode introduces a Fresnel reflection.

According to the design of compact focusing grating couplers (FGCs) [3], an array of elliptical grating lines (see in Fig. 1(a)) can be used to couple light between a single-mode waveguide and a single mode fiber. A normal FGC 1(b) uses the part of the confocal ellipses with the azimuthal angle of the entrance waveguide $\phi=0$. However, since the phase-matching condition of the curved grating is fulfilled for all parts of the ellipses, $\phi$ can be titled to a nonzero angle. In this case, light can be still coupled out into the same direction. But the on-chip reflections are refocused away from the entrance waveguide, which suppresses the on-chip retroreflection. Simulation results are reported in [4], and it was shown that around $1550 \mathrm{~nm}$, the reflection power from the tilted focusing grating coupler (TFGC) with $\phi=45^{\circ}$ is around $5 \mathrm{~dB}$ to $15 \mathrm{~dB}$ lower (for different frequencies) compared to that of the normal FGC.

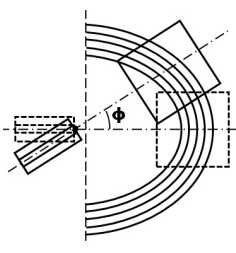

(a)

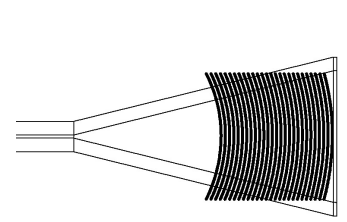

(b)

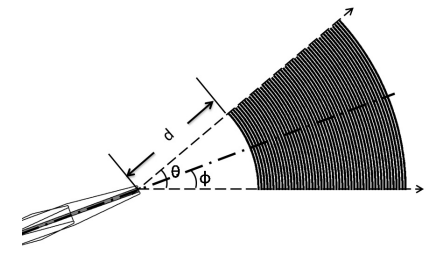

(c)

Fig. 1. 1(a): Schematic illustration of the ellipses used in focusing grating couplers (FGCs) on SOI. The dashed box shows the part used by a normal FGC. In our proposal, the grating in the box with a nonzero azimuthal angle $\phi$ is used. 1(b): a normal FGC. 1(c): a TFGC with an azimuthal angle $\phi=20^{\circ}$ and an opening angle of $\theta=40^{\circ}$.

\section{Designs, measurements and discussions}

A TFGC on SOI with an azimuthal angle $\phi=20^{\circ}$ was designed (shown in Fig. 1(c)) and fabricated with $193 \mathrm{~nm}$ DUV lithography in imec, Belgium. The top silicon layer had a thickness of $220 \mathrm{~nm}$, and the curves in the grating 
region were shallowly etched trenches with a silicon thickness of $150 \mathrm{~nm}$. No top cladding covered the grating. The distance from the entrance waveguide to the first grating $(d)$ was $24.8 \mu \mathrm{m}$, and the opening angle of the grating $(\theta)$ was $40.0^{\circ}$ (see in Fig. 1(c)). To reduce reflections, the deeply etched entrance waveguide was tapered to a shallowly etched aperture. The width of the aperture was set as $0.9 \mu \mathrm{m}$ to make sure that the light reaching the grating is mode matched with the mode in the single mode fiber. The entrance waveguide was connected to a standard grating coupler from the left side via a $2 \mathrm{~mm}$ long taper (see in Fig 2). A normal FGC with the same input grating coupler and taper was fabricated to compare with the TFGC (see in Fig. 2). The separation $L$ between the normal grating coupler and the input grating coupler was around $2.04 \mathrm{~mm}$. The four bends with $5 \mu \mathrm{m}$ radii in the TFGC test structure were used to mark the distance $L$. Both grating couplers were designed to operate at $1.55 \mu \mathrm{m}$.

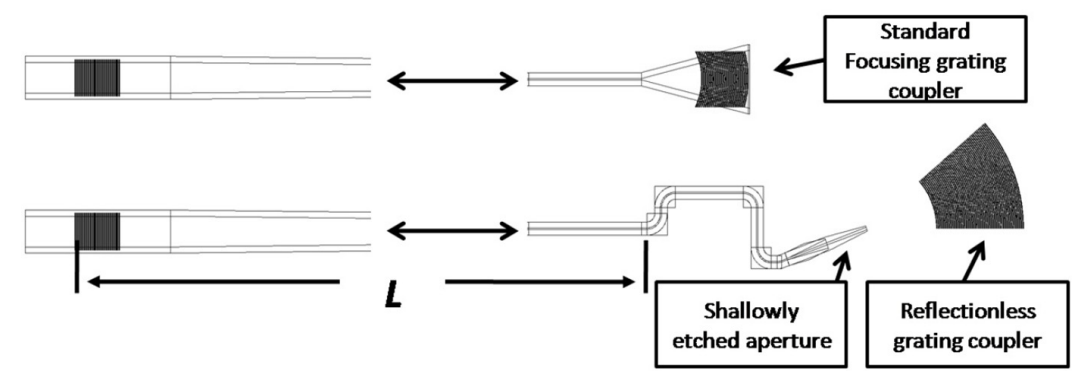

Fig. 2. Two different structures were measured: a normal FGC and a TFGC. Laser signals were sent from the left side through the standard grating couplers, and connected to the test grating couplers through a straight taper with a length of around $2 \mathrm{~mm}$.

Coherent light sweeping from $1490 \mathrm{~nm}$ to $1570 \mathrm{~nm}$ was sent into the input grating couplers via a single mode fiber pigtail. The sampling rate of the detector was set at a specific value to record a discrete spectrum with a wavelength step of $20 \mathrm{pm}$. The transmission maxima for both test grating couplers lay between $1540 \mathrm{~nm}$ and $1550 \mathrm{~nm}$ as expected. Their fiber-to-fiber insertion losses (around $-10 \mathrm{~dB}$ ) and $3 \mathrm{~dB}$ bandwidths (around $50 \mathrm{~nm}$ ) were also similar.

The reflections were measured by using the optical frequency domain reflectometry (OFDR) technique. With the help of a circulator [5], beating signals between the reflection from the input fiber/air interface and those from the on-chip device were detected. The output fiber pigtail was removed in the reflection measurements to avoid additional reflections. Reflections at different locations in the test structures can be extracted from the autocorrelation $R(z)$ of the detected signals. Three steps were done before calculating $R(z)$ : 1. mapping the reflection spectra from the wavelength domain to the frequency domain, using the linear interpolation function. 2. multiplying a Gaussian window to the reflections in the frequency domain, so as to suppress numerical leakages. 3. zero padding, in order to get a denser frequency mesh in the autocorrelation function, which is good for estimating the $3 \mathrm{~dB}$ bandwidth of peaks. After these steps, a power spectral density $P(f)$ for an array of frequencies $f$ was obtained. $R(z)$ was then derived by calculating the inverse Fourier transform of $P(f)$. The group index used in the calculation (3.75) was the average group index along the taper. A reference measurement was also made by shining the input light to an empty area (with no patterns) on the same chip, so that reflections from the backside of the chip can be extracted. In Fig. 3(a), the autocorrelations $R(z)$ from the three measurements are shown on a logarithmic scale $(20 \times \log (R(z)))$. Note that the calculated reflectance is an average value for wavelengths between $1490 \mathrm{~nm}$ and $1570 \mathrm{~nm}$.

The strong reflections from different locations in the test structures formed beating signals on the detector by interfering with the reflection from the fiber/air interface, and those strong beatings were represented by the maximal values in the $R(z)$. The first maximum near $z=15 \mu \mathrm{m}$ (in Fig.3(b)) may be caused by the beating between the reflection from the fiber/air interface and that from the input grating coupler. The second important maximal value appear for $z \approx 0.71 \mathrm{~mm}$ (in Fig. 3(c)), which is found in all $R(z)$ curves. It is cause by the beating between the reflection from the fiber/air interface and that from the backside of the chip. The peak for $z>2 \mathrm{~mm}$ (marked by arrows in Fig. 3(d)) is caused by the beating between the reflection from the fiber/air interface and that from the test grating couplers (output grating couplers), and thus it does not appear in the autocorrelation of the reference measurement. It can be found that the peak of the TFGC is $0.15 \mathrm{~mm}$ further away than that of the normal FGC, which is because the TGGC lies to the right of the normal FGC (see in Fig. 2). Compared to the peak of the normal FGC, that of the TFGC is lower. Reflectance values integrated over the $3 \mathrm{~dB}$ linewidth of the peaks illustrate that the average reflectance of the TFGC is $9.5 \mathrm{~dB}$ less than that of the normal FGC. Reflectance from the four bends in the TFGC test structure is also quite large 


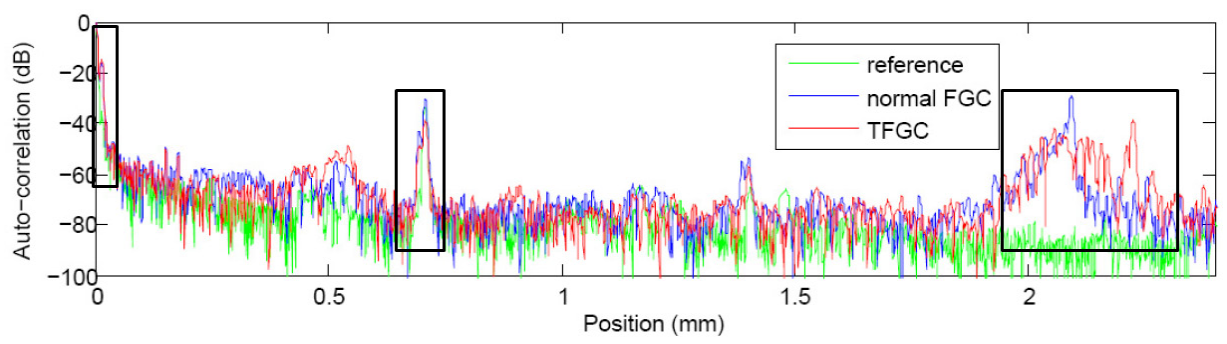

(a)

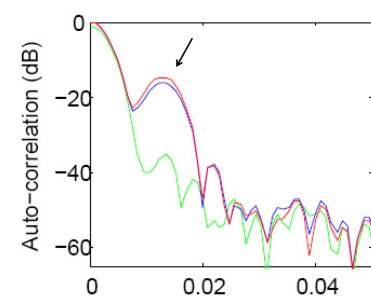

(b)

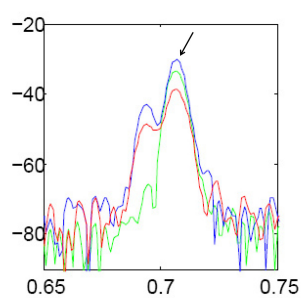

(c)

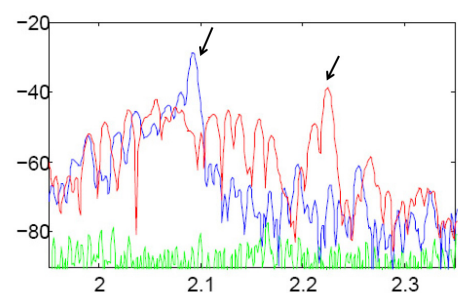

(d)

Fig. 3. 3(a): Autocorrelation of reflections from three test structures: a normal focusing grating coupler (FGC), a tilted focusing grating coupler (TFGC), and an empty area (reference). Three important reflections are shown: 3(b) reflections from the input grating coupler, 3(c) reflections from the chip backside, and 3(d) reflections from the output grating couplers.

as is shown in Fig. 3(d), but can be easily avoided in a proper design.

\section{Conclusions}

In the tilted focusing grating coupler (TFGC) with an azimuthal angle of $20^{\circ}$, the average return loss between $1490 \mathrm{~nm}$ and $1570 \mathrm{~nm}$ is $9.5 \mathrm{~dB}$ better than that of the normal focusing grating coupler (FGC), while its insertion loss is not reduced. According to simulations [4], increasing the angle will further increase the return losses. It is also shown that strong reflections can be introduced by sharp bends in deeply etched waveguides.

\section{Acknowledgements}

Y. Li acknowledge the Methusalem grant "smart photonic chips"from the Flemish government and ePIXfab for the fabrication of the chip. D. Vermeulen thanks the Institute for the Promotion of Innovation by Science and Technology in Flanders (IWT) for a grant. Y. De Koninck acknowledges the Fund for Scientific Research (FWO-Vlaanderen) for a PhD grant.

\section{References}

1. D.Vermeulen, S. Selvaraja, Pl.Verheyen, G. Lepage, W. Bogaerts, P. Absil, D. Van Thourhout, and G. Roelkens, "High-efficiency fiber-to-chip grating couplers realized using an advanced CMOS-compatible Silicon-OnInsulator platform," Optics Express 18, 18278-18283 (2011).

2. D. Taillaert, P. Bienstman, and R. Baets, "Compact efficient broadband grating coupler for silicon-on-insulator waveguides," Optics letters, 29, 2749-2751 (2004).

3. F. Van Laere, T. Claes, J. Schrauwen, S. Scheerlinck, W. Bogaerts, D. Taillaert, L. O'Faolain, D. Van Thourhout, and R. Baets, "Compact Focusing Grating Couplers for Silicon-on-Insulator Integrated Circuits," IEEE Photonics Technology Letters, 19, 1919-1921 (2007).

4. D. Vermeulen, Y. De Koninck, Y. Li, E. Lambert, W. Bogaerts, R. Baets, G. Roelkens, "Reflectionless Grating Coupling for Silicon-on-Insulator Integrated Circuits", Group IV Photonics (London), 74-76 (P1.6) (2011)

5. F. Morichetti, A. Canciamilla, C. Ferrari, M. Torregiani, A. Melloni, and M. Martinelli, "Roughness Induced Backscattering in Optical Silicon Waveguides”, Physical Review Letters, 104, 033902 (2010) 